\title{
Linear Complexity Profiles and Continued Fractions
}

\author{
Muzhong Wang \\ Department of Electrical Engineering \\ University of Waterloo \\ Waterloo, Ontario, Canada N2L 3G1
}

\begin{abstract}
The linear complexity, $\mathcal{L}\left(s^{n}\right)$, of a sequence $s^{n}$ is defined as the length of the shortest linear feedback shift-register (LFSR) that can generate the sequence. The linear complexity profile, $L_{4^{n}}=L_{1} L_{2} \ldots L_{n}$, of $s^{n}$ (where $L_{i}=\mathcal{L}\left(s^{i}\right), 1 \leq i \leq n$, denotes the linear complexity of first $i$ digits of $s^{n}$ ) provides better insight into the complexity of an individual sequence. By the increment sequence $\Delta_{, n}=\Delta_{1} \Delta_{2} \cdots \Delta_{m}$ in a linear complexity profile, $L_{1} L_{2} \ldots L_{n}$, we mean the subsequence of positive numbers in the sequence $L_{1}\left(L_{2}-L_{1}\right) \ldots\left(L_{n}-L_{n-1}\right)$. For example, if $L_{1} \cdots L_{5}=02223$, its increment sequence is $\Delta_{, 5}=\Delta_{1} \Delta_{2}=21$. If we associate a sequence $s^{n}$ over $F$ with an element $S(z)$ in the field of Laurent series over $F$ in the following way

$$
s^{n}=s_{1} s_{2} \cdots s_{n} \Longleftrightarrow S(z)=s_{1} z^{-1}+s_{2} z^{-2}+\cdots+s_{n} z^{-n},
$$

$S(z)$ can then be written as

$$
S(z)=a_{0}(z)+\frac{1}{a_{1}(z)+\frac{1}{a_{2}(z)+\frac{1}{a_{k}(z)}}},
$$

where $a_{i}(z) \in F[z]$, the ring of polynomials in $z$ over $F$, for all $i \geq 0$. It will be shown that, for a sequence $s^{n}$, the increment sequence $\Delta s^{n}$ of the linear complexity profile of $s^{n}$ is as follows. (1) If $2 \cdot \sum_{i=1}^{k} \operatorname{deg}\left(a_{i}(z)\right)-\operatorname{deg}\left(a_{k}(z)\right) \leq n$, then $\Delta_{n^{n}}=$ $\operatorname{deg}\left(a_{1}(z)\right) \operatorname{deg}\left(a_{2}(z)\right) \cdots \operatorname{deg}\left(a_{k}(z)\right)$. (2) If $2 \cdot \sum_{i=1}^{k} \operatorname{deg}\left(a_{i}(z)\right)-\operatorname{deg}\left(a_{k}(z)\right)>n$, then $\Delta_{, n}=\operatorname{deg}\left(a_{1}(z)\right) \operatorname{deg}\left(a_{2}(z)\right) \cdots \operatorname{deg}\left(a_{k^{\prime}}(z)\right)$, where $k^{\prime}=\max \left\{j: 2 \cdot \sum_{i=1}^{j} \operatorname{deg}\left(a_{i}(z)-\right.\right.$ $\left.\operatorname{deg}\left(a_{j}(z)\right) \leq n\right\}$.
\end{abstract}




\section{Introduction}

It has long been known that there is some sort of connection between linear complexity concepts and continued-fraction theory Recently, H. Niederreiter has done lots of works on the problem [NIED 87] [NIED 88] [NIED 89]. If sequences are associated with the elements in the field of Laurent series, the linear complexity profile of a sequence is totally specified by the degrees of partial quotients in the continued-fraction expansion of the corresponding Laurent series [NIED 87]. We will prove that the sequence of "jumps" in the linear complexity profile of a sequence is equal to the sequence of degrees of partial quotients in the continued-fraction expansion of the corresponding Laurent series. Therefore, sequences with desired linear complexity profiles can be constructed by choosing the degrees of partial quotients in the continued-fraction expansion.

We first give a short introduction to continued-fraction expansions in the field of Laurent series (Laurent series field). A Laurent series in the indeterminate $z$ over the field $F$ is an expression of the form

$$
f_{l}(z)=\sum_{j=-\infty}^{+\infty} a_{j} z^{j}
$$

for which $a_{j} \in F$, all $j$, and where $a_{j}=0$ for $j>d$, where $d$ is some integer. The degree of $f_{l}(z)$, denoted by $\operatorname{deg} f_{l}(z)$, is the largest $j$ (if any) such that $a_{j} \neq 0$ and is, by way of convention, $-\infty$ when $a_{j}=0$ for all $j$. For instance, the Laurent series $z+1+z^{-1}+z^{-2}+\ldots$ has degree 1 whereas the Laurent series $z^{-1}+z^{-2}+z^{-3}+\ldots$ has degree -1 . Addition and multiplication of Laurent series is defined in the same way as for power series. The set of all Laurent series in $z$ over the field $\boldsymbol{F}$ forms a field that we denote by $\boldsymbol{F}\left(z^{-1}\right)$. A polynominal is a Laurent series for which $a_{j}=0$ for all $j<0$. Note that the ring of polynomials in $z$ over $\boldsymbol{F}$, denoted by $\boldsymbol{F}[\boldsymbol{z}]$, is a subring of the field $\boldsymbol{F}\left(z^{-1}\right)$.

For a Laurent series $f_{l}(z)$, one defines its valuation, $\left\|f_{i}(z)\right\|$, by

$$
f_{l}(z)=\left\{\begin{array}{lll}
2^{\operatorname{deg}} f_{l}(x) & \text { if } & f_{l}(z) \neq 0 \\
0 & \text { if } & f_{l}(z)=0
\end{array}\right.
$$

This is a nonarchimedean valuation because

$$
\left\|f_{l}(z)+g_{l}(z)\right\| \leq \max \left\{\left\|f_{l}(z)\right\|,\left\|g_{l}(z)\right\|\right\}
$$


which is stronger than the more usual "norm inequality" in which the right side is the sum of the two valuations.

For convenience, we summarize without proof some obvious properties of $\|$.$\| .$

Lemma $1 \|$ || has the following properties:

P1. $\left\|f_{l}(z) g_{l}(z)\right\|=\left\|f_{l}(z)\right\|\left\|g_{l}(z)\right\|$.

P2. $\left\|f_{l}(z)\right\| \geq 0$ with equality if and only if $f_{l}(z)=0$.

P3. $\left\|f_{l}(z)+g_{l}(z)\right\| \leq \max \left\{\left\|f_{l}(z)\right\|,\left\|g_{l}(z)\right\|\right\}$, with equality if $\left\|f_{l}(z)\right\| \neq\left\|g_{l}(z)\right\|$.

P4. $\|1\|=1$.

P5. $\left\|f_{l}(z)^{-1}\right\|=\left\|f_{l}(z)\right\|^{-1}$.

P6. $\left\|-f_{l}(z)\right\|=\left\|f_{l}(z)\right\|$.

P7. If $f_{l}(z) \in \boldsymbol{F}[z]$ and $f_{l}(z) \neq 0$, then $\left\|f_{l}(z)\right\| \geq 1$.

Euclid's division theorem for polynomials can be restated in terms of $\|\cdot\|$ as follows.

Theorem 1 (Euclid's Division Theorem for Polynomials) If $f(z)$ and $g(z)$ are in $F[z]$ with $g(z) \neq 0$, then there exists unique $q(z)$ and $r(z)$ in $F[z]$ such that

$$
f(z)=q(z) g(z)+r(z) \quad \text { and }\|r(z)\|<\|g(z)\| .
$$

A continued-fraction in the indeterminate $z$ over the field of $F$ is an expression of the form

$$
a_{0}(z)+\frac{1}{a_{1}(z)+\frac{1}{a_{2}(z)+}},
$$

where $a_{i}(z) \in \boldsymbol{F}[z]$ for all $i \geq 0$ and either (1) $\operatorname{deg} a_{i}(z) \geq 1\left(\left\|a_{i}(z)\right\| \geq 2\right)$ for all $i \geq 1$ (in which case the continued-fraction is called to be infinite) or (2), for some positive integer $N$, 
$\operatorname{deg} a_{i}(z) \geq 1$ for $1 \leq i \leq N$ and $a_{i}(z)=0$ for all $i>N$ (in which case the continued-fraction is said to be finite). The polynomials $a_{i}(z)$ are called the partial quotients of the continuedfraction. There is a unique way in which the indicated divisions in a continued-fraction can be carried out to give a Laurent series, and we thus regard hereafter a continued-fraction as an element of $\boldsymbol{F}\left(z^{-1}\right)$.

Given any continued-fraction, let $\left[a_{0}(z) ; a_{1}(z), \ldots, a_{n}(z)\right]$ denote the finite continuedfraction obtained by setting $a_{i}(z)=0$ for all $i \geq n$, i.e., the finite continued-fraction

$$
a_{0}(z)+\frac{1}{a_{1}(z)+\frac{1}{a_{2}(z)+\quad} \cdot \frac{1}{a_{i}(z)}} .
$$

Every finite continued-fraction can, after clearing of denominators, be written as the ratio of two polynomials, i.e., as an element of $\boldsymbol{F}(z)$, the field of rational functions over $\boldsymbol{F}$. Thus one can write

$$
\left[a_{0}(z) ; a_{1}(z), \cdots, a_{n}(z)\right]=\frac{p_{n}(z)}{q_{n}(z)}, \quad n \geq 0,
$$

where $p_{n}(z)$ and $q_{n}(z)$ are polynomials, defined recursively by

$$
\begin{array}{ll}
p_{0}(z)=a_{0}(z), & p_{k}(z)=a_{k}(z) p_{k-1}(z)+p_{k-2}(z)(k \geq 1), \\
q_{0}(z)=1, & q_{k}(z)=a_{k}(z) q_{k-1}(z)+q_{k-2}(z)(k \geq 1)
\end{array}
$$

where, by way of convention, $p_{-1}(z)=1$ and $q_{-1}(z)=0$. The rational function $\frac{p_{n}(z)}{q_{n}(z)}$ is called the $n$-th convergent of the continued-fraction $\left[a_{0}(z) ; a_{1}(z), \cdots, a_{i}(z), \ldots\right]$.

The following lemma is proved in [LID-NIE 83, pp.235-239].

Lemma 2 The convergents of $\left[a_{0}(z) ; a_{1}(z), \ldots, a_{i}(z), \ldots\right]$ have the following properties:

$$
p_{k}(z) q_{k-1}(z)-p_{k-1}(z) q_{k}(z)=(-1)^{k-1} \quad(k \geq 1)
$$

or, equivalently,

$$
\frac{p_{k}(z)}{q_{k}(z)}-\frac{p_{k-1}(z)}{q_{k-1}}=\frac{(-1)^{k-1}}{q_{k}(z) q_{k-1}(z)} \quad(k \geq 1) .
$$


Equation (3) implies that

$$
\operatorname{gcd}\left(p_{i}(z), q_{i}(z)\right)=1 \quad \text { for } i \geq 1
$$

The following property of convergents, which appears to be new, will play an important role in the sequel.

Lemma 3 The denominator $q_{n}(z)$ of the $n$-th convergent to $\left[a_{0}(z) ; a_{1}(z), \ldots, a_{i}(z), \ldots\right]$ satisfies

$$
\begin{aligned}
\left\|q_{0}(z)\right\| & =1 \\
\left\|q_{n}(z)\right\| & =\prod_{j=1}^{n}\left\|a_{j}(z)\right\|, \quad n \geq 1
\end{aligned}
$$

provided $a_{n}(z) \neq 0$.

Proof. Because $q_{0}(z)=1$, we have $\left\|q_{0}(z)\right\|=1$ as claimed. Because

$$
q_{1}(z)=a_{1}(z),
$$

(6) holds trivially for $n=1$.

Suppose that (6) holds for $1 \leq n \leq N$. Because $\left\|a_{j}(z)\right\|>1$ for $1 \leq j \leq N,\left\|q_{N-1}(z)\right\|$ is strictly smaller than $\left\|q_{N}(z)\right\|$. Thus

$$
\begin{aligned}
\left\|q_{N+1}(z)\right\| & =\left\|a_{N+1}(z) q_{N}(z)+q_{N-1}(z)\right\| \\
& =\left\|a_{N+1}(z) q_{N}(z)\right\| \quad \text { (P3 in Lemma 1) } \\
& =\left\|a_{N+1}(z)\right\| \cdot\left\|q_{N}(z)\right\| \quad \text { (P1 in Lemma 1) } \\
& =\prod_{j=1}^{N+1}\left\|a_{j}(z)\right\| .
\end{aligned}
$$

This completes the proof by induction.

The following theorem, which is proved in [WES-SCH 79, theorem 2], shows the sense in which the $n$-th convergent is the best approximation to

$$
\left[a_{0}(z) ; a_{1}(z), \ldots, a_{i}(z), \ldots\right]=S(z) .
$$


Theorem 2 The convergents to $\left[a_{0}(z) ; a_{1}(z), \ldots, a_{i}(z), \ldots\right]$ have the property that, for every $n(n \geq 0)$, if $q(z)$ is a polynomial with $\|q(z)\|<\left\|q_{n+1}(z)\right\|$, then, for any polynomial $p(z)$ such that

it must hold that

$$
\frac{p(z)}{q(z)} \neq \frac{p_{n}(z)}{q_{n}(z)}
$$

$$
\left\|\frac{p_{n}(z)}{q_{n}(z)}-S(z)\right\|<\left\|\frac{p(z)}{q(z)}-S(z)\right\| .
$$

Let $g_{l}(z)$ be an element in $\boldsymbol{F}\left(z^{-1}\right)$. If also $g_{l}(z) \in \boldsymbol{F}(z)$, then

$$
g_{l}(z)=\frac{r_{-2}(z)}{r_{-1}(z)}
$$

where $r_{-2}(z)$ and $r_{-1}(z)$ are polynomials with $\left\|r_{-1}(z)\right\| \geq 1$. There exist unique polynomials $a_{0}(z)$ and $r_{0}(z)$ such that $r_{-2}(z)=a_{0}(z) r_{-1}(z)+r_{0}(z)$ and $\left\|r_{0}(z)\right\|<\left\|r_{-1}(z)\right\|$. Equivalently,

$$
g_{l}(z)=a_{0}(z)+\frac{r_{0}(z)}{r_{-1}(z)} .
$$

If $\left\|r_{0}(z)\right\| \neq 0$, then by the same argument there exist unique polynomials $a_{1}(z)$ and $r_{1}(z)$ such that

$$
\frac{r_{-1}(z)}{r_{0}(z)}=a_{1}(z)+\frac{r_{1}(z)}{r_{0}(z)}
$$

and $\left\|r_{1}(z)\right\|<\left\|r_{0}(z)\right\|<\left\|r_{-1}(z)\right\|$. Continuing in this manner, we must eventually reach the case $r_{N}(z)=0$ because the degrees of $r_{-1}(z), r_{0}(z), r_{1}(z), \cdots$ are strictly decreasing. Thus it follows that we can always write a rational function $\frac{r_{-2}(z)}{r_{-1}(z)}$ as a finite continued-fraction

$$
\frac{r_{-2}(z)}{r_{-1}(z)}=\left[a_{0}(z) ; a_{1}(z), \cdots, a_{N}(z)\right]
$$

The converse statement that every finite continued-fraction $\left[a_{0}(z) ; a_{1}(z), \ldots, a_{N}(z)\right]$ represents an element of $\boldsymbol{F}(\boldsymbol{z})$ was noted previously.

$$
\text { Example. } \quad \begin{aligned}
r_{2}(z) & =z^{3}+z^{2}+1, \quad r_{-1}(z)=z^{4} \\
a_{0}(z) & =0 \\
r_{0}(z) & =z^{3}+z^{2}+1 \\
a_{1}(z) & =z+1, \quad r_{1}(z)=z^{2}+z+1,
\end{aligned}
$$




$$
\begin{aligned}
a_{2}(z) & =z, \quad r_{2}(z)=z+1, \\
a_{3}(z) & =z, \quad r_{3}(z)=1, \\
a_{4}(z) & =z+1, \quad r_{4}(z)=0, \\
\frac{r_{-2}(z)}{r_{-1}(z)} & =\frac{z^{3}+z^{2}+1}{z^{4}} \\
& =\frac{1}{z+1+\frac{1}{z+\frac{1}{z+\frac{1}{z+1}}}} \\
& =[0 ; z+1, z, z, z+1] .
\end{aligned}
$$

\section{Relation between Linear Complexity Profile and Continued Fractions}

The linear complexity $\mathcal{L}\left(s^{n}\right)$ of a sequence, $s^{n}=s_{1} s_{2} \cdots s_{n}$ where $s_{1}, s_{2}, \ldots, s_{n}$ are from a field $\boldsymbol{F}$, can also be defined as the smallest nonnegative integer $\mathrm{L}$ such that there exist $c_{0}, c_{1}, \cdots, c_{L}$ in $\boldsymbol{F}$ satisfying

$$
c_{L} s_{i+L}+c_{L-1} s_{i+L-1}+\cdots+c_{0} s_{i}=0,1 \leq i \leq n-L
$$

where $c_{L} \neq 0$. The monic polynomial $c_{L}^{-1}\left(c_{L} D^{L}+\ldots+c_{1} D+c_{0}\right)$ is called a characteristic polynomial of the sequence; we remark that the characteristic polynomial is unique if and only if $L \leq n / 2$.

The linear complexity profile $L, n$ is defined as the sequence

$$
L_{, n}=L_{1} L_{2} \cdots L_{n}
$$

where $L_{i}=\mathcal{L}\left(s^{i}\right)$. The definition of linear complexity implies

$$
L_{i} \geq L_{j} \quad \text { for } i>j
$$

We associate a sequence $s^{n}$ over $\boldsymbol{F}$ with an element $S(z)$ in the field of Laurent series over $\boldsymbol{F}$ in the following way

$$
s^{n}=s_{1} s_{2} \cdots s_{n} \Longleftrightarrow S(z)=s_{1} z^{-1}+s_{2} z^{-2}+\cdots+s_{n} z^{-n} .
$$


We see immediately that the sequence $s^{n}$ and the sequence $s^{n+m}=s^{n} 0^{m}$ (where $s^{n} 0^{m}$ denotes the concatenation of $s^{n}$ and $0^{m}$ ) are associated with the same element $\sum_{i=1}^{n} s_{i} z^{-i}$ in the field of Laurent series. Therefore, we can implicitly expand $s^{n}=s_{1} s_{2}, \cdots s_{n}$ to a semi-infinite sequence $s^{\infty}$ by concatenating $s^{n}$ with infinitely many zeroes,

$$
s^{\infty}=s_{1} s_{2} \cdots s_{n} 000 \cdots
$$

Suppose that $S(z)=s_{1} z^{-1}+s_{2} z^{-2}+\cdots$ is a Laurent series with $\|S(z)\|<1$. Letting $q(z)=c_{L} z^{L}+\cdots+c_{1} z+c_{0}, c_{L} \neq 0$, we see that the left side of (7) is the coefficient of $z^{-i}$ in the product $S(z) q(z)$. Thus, if $(7)$ holds, there is a unique polynomial $p(z)$ such that $\| p(z)-S(z) q(z)) \|<2^{n+L}$ and hence (by P1 and P5) such that

$$
\left\|\frac{p(z)}{q(z)}-S(z)\right\|<2^{-n} .
$$

Moreover, $\|p(z)\|<\|q(z)\|=2^{L}$.

Conversely, if (10) holds where $q(z)=c_{L} z^{L}+\cdots+c_{1} z+c_{0}$ and $p(z)$ are polynomials with $\|q(z)\|=2^{L}$, then (7) also holds and $\|p(z)\|<\|q(z)\|$. We have thus proved the following lemma.

Lemma 4 The linear complexity of a sequence $s^{n}=s_{1} s_{2} \ldots s_{n}$ is equal to the minimum degree of polynomials $q(z)$ such that there exists a polynomial $p(z)$ satisfying

$$
\left\|\frac{p(z)}{q(z)}-S(z)\right\|<2^{-n},
$$

where $S(z)=s_{1} z^{-1}+s_{3} z^{-2}+\cdots+s_{n} z^{-n}$. Moreover, $c_{L}^{-1} q(z)$ is a characteristic polynomial of $s^{n}$, where $c_{L}$ is the leading coefficient of $q(z)$.

Because $S(z)$ is in $F(z), S(z)$ can be expressed as a finite continued-fraction

$$
\begin{aligned}
S(z) & =a_{0}(z)+\frac{1}{a_{1}(z)+\frac{1}{a_{2}(z)+}} \\
& =\left[0 ; a_{1}(z), \cdots, a_{N}(z)\right]
\end{aligned}
$$


for some $N$ (where the polynomial part vanishes because $\|S(z)\|<1$ ). Notice that $a_{0}(z)$ is always zero.

The following theorem is proved in [NIED 87]. For readers' convenience, we give an alternative proof here.

Theorem 3 The linear complexity profile, $L, \infty$, of the sequence $s^{\infty}$ is totally specified by the degrees of the partial quotients in the continued-fraction expansion of $S(z)$, in the following way:

$L 1$ if $\operatorname{deg} a_{1}(z)>1, L_{i}=0,1 \leq i<\operatorname{deg} a_{1}(z)$;

$$
\begin{aligned}
& L 2 L_{i}=\operatorname{deg} a_{1}(z), \operatorname{deg} a_{1}(z) \leq i<\operatorname{deg} a_{2}(z)+\operatorname{deg} a_{1}(z) ; \\
& L_{i}=\operatorname{deg} a_{2}(z)+\operatorname{deg} a_{1}(z), \operatorname{deg} a_{2}(z)+2 \operatorname{deg} a_{1}(z) \leq i<\operatorname{deg} a_{3}(z)+2 \sum_{i=1}^{2} \operatorname{deg} a_{i}(z) ; \\
& \quad \vdots \\
& L_{i}=\sum_{i=1}^{N-1} \operatorname{deg} a_{i}(z), \operatorname{deg} a_{N-1}(z)+2 \sum_{i=1}^{N-2} \operatorname{deg} a_{i}(z) \leq i<\operatorname{deg} a_{N}(z)+2 \sum_{i=1}^{N-1} \operatorname{deg} a_{i}(z) ; \\
& \quad \vdots
\end{aligned}
$$

Proof. $\mathrm{L} 1$ is obvious because $\operatorname{deg} a_{1}(z)>1$ implies $s_{1}=\ldots=s_{\left(\operatorname{deg} a_{1}(z)-1\right)}=0$.

Consider the convergents

$$
\frac{p_{n}(z)}{q_{n}(z)}=\left[0 ; a_{1}(z), a_{2}(z), \cdots, a_{n}(z)\right], \quad n \geq 1 .
$$

We know from Lemma 2 and Lemma 3 that

$$
\frac{p_{n+1}(z)}{q_{n+1}(z)}-\frac{p_{n}(z)}{q_{n}(z)}=\frac{(-1)^{n}}{q_{n+1}(z) p_{n+1}(z)}
$$

and that $\xi_{n}=\operatorname{deg}\left[q_{n}(z) q_{n+1}(z)\right]=\operatorname{deg} a_{n+1}(z)+2 \sum_{i=1}^{n} \operatorname{deg} a_{i}(z)$. This implies that the coefficients of $z^{-i}$ for $1 \leq i<\xi_{n}$ in the Laurent series for $\frac{p_{n+1}(z)}{q_{n+1}(z)}$ and $\frac{p_{n}(z)}{q_{n}(z)}$ are the same but that the coefficients of $z^{t_{n}}$ are different. Thus

$$
\frac{p_{n}(z)}{q_{n}(z)}=s_{1}^{\prime} z^{-1}+\cdots+s_{\xi_{n}-1}^{\prime} z^{-\left(\xi_{n}-1\right)}+s_{\xi_{n}}^{\prime} z^{-\xi_{n}}+\cdots
$$


where

$$
\begin{aligned}
& s_{i}^{\prime}=s_{i} \quad \text { for } 1 \leq i \leq \xi_{n}-1, \text { and } \\
& s_{\xi_{n}}^{\prime} \neq s_{\xi_{n}} .
\end{aligned}
$$

We have then

$$
\left\|\frac{p_{n}(z)}{q_{n}(z)}-S(z)\right\|=2^{-\xi_{n}}
$$

According to Lemma 4,

$$
\mathcal{L}\left(s_{1} s_{2} \cdots s_{\xi_{n}}\right) \geq \operatorname{deg} q_{n}(z) .
$$

By the same argument that gives (11), we have

$$
\left\|\frac{p_{n+1}(z)}{q_{n+1}(z)}-S(z)\right\|=2^{-\xi_{(n+1)}} .
$$

Theorem 4 shows that there exists no polynomials $p(z)$ and $q(z)$ with $\|q(z)\|<\left\|q_{n+1}(z)\right\|$ such that

$$
\left\|\frac{p(z)}{q(z)}-S(z)\right\|<\left\|\frac{p_{n}(z)}{q_{n}(z)}-S(z)\right\| .
$$

That is to say, $q_{n+1}(z)$ is the polynomial with minimum degree such that (13) holds.

It now follows from Theorem 2, (12) and (13) that

$$
\mathcal{L}\left(s_{1} s_{2} \ldots s_{i}\right)=\operatorname{deg} q_{n+1}(z) \text { for } \xi_{n} \leq i<\xi_{n+1} .
$$

This proves $\mathrm{L} 2$.

By the increment sequence $\Delta_{1} \Delta_{2} \cdots \Delta_{m}$ in a linear complexity profile, $L_{1} L_{2} \cdots L_{n}$, we mean the subsequence of positive numbers in the sequence $L_{1}\left(L_{2}-L_{1}\right) \ldots\left(L_{n}-L_{n-1}\right)$. For example, if $L_{1} \cdots L_{5}=02223$, its increment sequence is $\Delta_{1} \Delta_{2}=21$.

Lemma 5 The linear complexity profile $L_{1} L_{2} \ldots L_{n}$ is uniquely determined by its increment sequence, and conversely.

Proof. The linear complexity profile trivially determines the increment sequence. The increment sequence uniquely determines the linear complexity after the $k$-th jump as $\Delta_{1}+\Delta_{2}+$ $\cdots+\Delta_{k}$. Suppose this jump occurs at position $i+1$, i.e., $L_{i+1}=\Delta_{1}+\Delta_{2}+\cdots+\Delta_{k}>L-i=$ 
$\Delta_{1}+\Delta_{2}+\cdots+\Delta_{k-1}$. By the "Length-Change Property of LFSR's" proved in [MASS 69, theorem 2], $L_{i+1} \neq L_{i}$ implies $L_{i+1}=i+1-L_{i}$ for all $i>0$ ( $L_{0}=0$ by way of convention). Thus

$$
\begin{aligned}
i+1 & =L_{i+1}+L_{i} \\
& =2 L_{i}+\left(L_{i+1}-L_{i}\right) \\
& =2\left(\Delta_{1}+\Delta_{2}+\cdots+\Delta_{k-1}\right)+\Delta_{k} .
\end{aligned}
$$

Thus, the location $i+1$ of the $k$-th jump $\left(L_{i+1}-L_{i}\right)$ is also uniquely determined by the increment sequence. This proves the lemma.

For instance, suppose that the increment sequence of a linear complexity profile is 132 , the linear complexity profile can only be

$$
L, \infty=1^{4} 4^{4} 6^{\infty} .
$$

With the aid of Lemma 5, we now have our main result.

Corollary 1 to Theorem 3. If a semi-infinite sequence $s^{\infty}=s_{1} s_{2} \cdots$ over a field $F$ is associated with the element $S(z)=\sum_{i=1}^{\infty} s_{i} z^{-i}$ in the field of Laurent series over $F$, then the increment sequence of the linear complexity profile of $s^{\infty}$ is equal to the sequence of degrees of the partial quotients in the continued-fraction expansion of $S(z)$, i.e., $\Delta_{k}=\operatorname{deg}\left[a_{k}(z)\right]$.

Corollary 2 to Theorem 3. If a finite sequence $s^{n}=s_{1} s_{2} \cdots s_{n}$ over a field $F$ is associated with the element $S(z)=\sum_{i=1}^{n} s_{i} z^{-i}=\left[0 ; a_{1}(z), a_{2}(z), \cdots, a_{k}(z)\right]$ in the field of Laurent series over $F$, then the increment sequence $\Delta, n$ of the linear complexity profile of $s^{n}$ is as follows.

1. If $2 \cdot \sum_{i=1}^{k} \operatorname{deg}\left(a_{i}(z)\right)-\operatorname{deg}\left(a_{k}(z)\right) \leq n$, then $\Delta_{\mathbf{t}^{n}}=\operatorname{deg}\left(a_{1}(z)\right) \operatorname{deg}\left(a_{2}(z)\right) \cdots \operatorname{deg}\left(a_{k}(z)\right)$.

2. If $2 \cdot \sum_{i=1}^{k} \operatorname{deg}\left(a_{i}(z)\right)-\operatorname{deg}\left(a_{k}(z)\right)>n$, then $\Delta_{z^{n}}=\operatorname{deg}\left(a_{1}(z)\right) \operatorname{deg}\left(a_{2}(z)\right) \cdots \operatorname{deg}\left(a_{k^{\prime}}(z)\right)$, where $k^{\prime}=\max \left\{j: 2 \cdot \sum_{i=1}^{j} \operatorname{deg}\left(a_{i}(z)-\operatorname{deg}\left(a_{j}(z)\right) \leq n\right\}\right.$.

These corollaries tell us how to construct (finite and infinite) sequences with desired linear complexity profiles.

Example. Construct all sequences over $\boldsymbol{F}_{2}$ that have the linear complexity profile $1^{4} 4^{4} 6^{\infty}$ of (17). The increment sequence of this linear complexity profile is 132 . According 
to the Corollary 1 to Theorem 3, a sequence with this increment sequence has the finite continued-fraction

$$
S(z)=\frac{1}{a_{1}(z)+\frac{1}{a_{2}(z)+\frac{1}{a_{3}(z)}}},
$$

where $\operatorname{deg} a_{1}(z)=1, \operatorname{deg} a_{2}(z)=3, \operatorname{deg} a_{3}(z)=2$. There are $2^{i}$ ways to choose a polynomial over $F_{2}$ with degree $i$. There are thus $2^{1} 2^{3} 2^{2}=64$ different choices for $S(z)$, i.e., there are 64 semi-infinite binary sequences having the linear complexity profile of (17). For a specific such sequence, we choose

$$
\begin{aligned}
& a_{1}(z)=z, \\
& a_{2}(z)=z^{3}, \\
& a_{3}(z)=z^{2},
\end{aligned}
$$

We have then

$$
\begin{aligned}
S(z) & =\frac{1}{z+\frac{1}{z^{3}+\frac{1}{z^{2}}}} \\
& =\frac{z^{6}+1}{z^{6}+z^{2}+z} .
\end{aligned}
$$

By long division, we find

$$
S(z)=z^{-1}+z^{-5}+z^{-9}+z^{-10}+\cdots .
$$

The desired sequence is

$$
s^{\infty}=1(000100011001010111110)^{\infty} .
$$

If a semi-infinite sequence $s^{\infty}$ corresponds to the element $S(z)$ of the Laurent field in the manner (9) such that the continued-fraction expansion is infinite, i.e.,

$$
S(z)=\left[0 ; a_{1}(z), \ldots, a_{k}(z), \ldots\right],
$$


then by using the $k$-th convergent of $S(z)$ to approximate $S(z)$, we can see that L1 and L2 in Theorem 3 still hold. If $k$ goes to infinity, the $k$-th convergent then approaches $S(z)$. Therefore, L1 and L2 in Theorem 3 and the Corollary 1 to Theorem 3 are also valid for the case that the continued-fraction expansion of $S(z)$ is infinite.

\section{Remarks}

In [NIED 86], Niederreiter showed the following result. If the continued-fraction expansion of $S(z)$ is infinite, which is the same as saying that $S(z)$ is irrational, the linear complexity profile satisfies

$$
\frac{1}{2}\left(i+1-K(S) \leq L_{i} \leq \frac{1}{2}(i+K(S)) \quad \text { for all } i \geq 1\right.
$$

where $L_{i}=\mathcal{L}\left(s_{1} s_{2} \cdots s_{i}\right)$ and $K(S)=\sup _{j>1} \operatorname{deg} a_{j}(z)$. We now show that (18) is a simple consequence of L2 in Theorem 3 and the "length-change property of LFSR's" for the case that the continued-fraction expansion of $S(z)$ is infinite.

We restate (2) in Theorem 3 as follows.

For

$$
\operatorname{deg} a_{k}(z)+2 \sum_{j+1}^{k-1} \operatorname{deg} a_{j}(z) \leq i \leq \operatorname{deg} a_{k+1}(z)+2 \sum_{j=1}^{k} \operatorname{deg} a_{j}(z)-1,
$$

where $k \geq 1$, we have

$$
\begin{aligned}
L_{i} & =\sum_{j=1}^{k} \operatorname{deg} a_{j}(z) \\
& =\frac{1}{2}\left(\operatorname{deg} a_{k}(z)+2 \sum_{j=1}^{k-1} \operatorname{deg} a_{j}(z)+\operatorname{deg} a_{k}(z)\right) \\
& \leq \frac{1}{2}\left(i+\operatorname{deg} a_{k}(z)\right)
\end{aligned}
$$

with equality when $i=\operatorname{deg} a_{k}(z)+2 \sum_{j=1}^{h-1}$, where the last step follows from the left inequality of (19).

Further,

$$
L_{i}=\sum_{j=1}^{k} \operatorname{deg} a_{j}(z)
$$




$$
=\frac{1}{2}\left(a_{k+1}(z)+2 \sum_{j=1}^{k} \operatorname{deg} a_{j}(z)-a_{k+1}(z)\right) .
$$

It follows then from the right inequality of (19) that

$$
L_{i} \geq \frac{1}{2}\left(i+1-\operatorname{deg} a_{k+1}(z)\right)
$$

with equality when $i=\operatorname{deg} a_{k+1}(z)+2 \sum_{j=1}^{k} a_{j}(z)-1$. Inequalities (22) and (23) immediately give (18).

Baum and Sweet [BAU-SWE 77] showed that all partial quotients of the continuedfraction expansion of $S(z)$ have degree one if and only if

$$
S^{2}(z)+z S(z)+1=(1+z) g^{2}(z)
$$

for some polynomial $g(z)$. Their equation (24) is the same as

$$
\begin{aligned}
s_{1} & =1, \quad \text { and } \\
s_{2 i+1} & =s_{2 i}+s_{i} \quad \text { for } i \geq 1
\end{aligned}
$$

The Corollary 1 to Theorem 3 implies then that all sequences $s^{\infty}$, for which $S(z)$ satisfies (24), have the linear complexity profile $112233 \ldots$, defined as the perfect linear complexity profile (PLCP). This is consistent with the result proved in [WAN-MAS 86], namely, that $s^{\infty}$ has a perfect linear complexity profile if and only if

$$
\begin{aligned}
s_{1} & =1 \\
s_{2 i+1} & =s_{2 i}+s_{i} \quad \text { for } i \geq 1 .
\end{aligned}
$$




\section{Acknowledgements}

I wish to thank Prof. James L. Massey for his guidance of this work. I further wish to thank Prof. Ian F. Blake for his encouragement and help.

\section{References}

[BAU-SWE 77] I. E. Baum and M. M. Sweet, "Badly approximable power series in characteristic 2", Ann. of Math. , 105 (1977), pp.573-580.

[LID-NIE 83] R. Lidl and H. Niederreiter, Finite Fields, Addison-Wesley Publishing Company, Inc., 1983.

[MASS 69] J. L. Massey, "Shift-Register Synthesis and BCH Decoding", IEEE Trans. on Info. Th., pp.122-127, IT-15, No.1, Jan. 1969.

[NIED 86] H. Niederreiter, "Continued Fractions for Formal Power Series, Pseudorandom Numbers, and Linear Complexity of Sequences", to appear in Contributions to General Algebra 5 (Proc. Conf. Salzburg, 1986), Teubner, Stuttgart.

[NIED 87] H. Niederreiter, "Sequences with almost perfect linear complexity profile", Proc. Eurocrypt'87, LNCS 304, 37-51 (1988).

[NIED 88] H. Niederreiter, "The probabilistic theory of linear complexity", Proc. Eurocrypt'88, LNCS 330, 191-209, (1988).

[NIED 89] H. Niederreiter, "Keystream sequence with good linear complexity profile for every starting point", paper presented at Eurocrypt's9, Hauthalen, Belgium.

[WAN-MAS 86] M. Z. Wang and J. L. Massey, "The Characterization of All Binary Sequences with Perfect Linear Complexity Profiles", paper presented at Eurocrypt'86, Linköping, Sweden.

[WES-SCH 79] L. R. Welch and R. A. Scholtz, "Continued Fractions and Berlekamp's Algorithm", IEEE Trans. on Info. Th., pp.19-27, IT-25, No.1, January 1979. 